\title{
Ready, shoot, aim? Summary justice for small hearts in nuclear cardiology
}

\author{
Guido Germano, PhD, MBA, ${ }^{\mathrm{a}, \mathrm{b}}$ and Paul B. Kavanagh, $\mathrm{MS}^{\mathrm{a}}$ \\ ${ }^{a}$ Department of Medicine, Cedars-Sinai Medical Center, Artificial Intelligence in Medicine \\ Program, Los Angeles \\ ${ }^{\mathrm{b}}$ David Geffen School of Medicine, UCLA, Los Angeles
}

Received May 5, 2016; accepted May 5, 2016

doi: 10.1007/s12350-016-0545-9

\section{See related article, pp. 1378-1388}

It may have happened to many of you to take a blurry photograph-maybe the subject moved unpredictably, or (as one of us has often experienced) the autofocus switch got dislodged as the camera was knocked around in the bag. If this was a mission-critical picture, playing around with sharpening filters and kernels in Photoshop will usually make things better, at times even serviceably so. Wouldn't it have been best to have focused appropriately to begin with, though? You bet it would have. As it turns out, things are not much different in nuclear medicine imaging.

It is well-known that nuclear cardiology (including SPECT and PET), while enjoying excellent image contrast due to differential radioisotopic uptake, does not count spatial image resolution as its strongest suit. This is directly related to the Nyquist-Shannon sampling theorem, stating that, in order to fully recover the maximum spatial frequency $f$ in an object (or, more generally, in a spatially varying signal), one must perform linear sampling at a frequency $f_{\mathrm{s}}$, where $f_{\mathrm{s}} \geq 2 f^{1}$ in practical terms, the distance between adjacent samples should be, at most, half the diameter of the smallest structure one wants to image. While the spatial resolution capabilities of standard SPECT cameras and PET systems can be theoretically very good, as evidenced by quality control tests routinely performed with bar phantoms, clinical considerations of patient dose, study

Reprint requests: G. Germano, Department of Medicine, Cedars-Sinai Medical Center, Artificial Intelligence in Medicine Program, Los Angeles; Guido.Germano@cshs.org

J Nucl Cardiol 2017;24:1389-92.

1071-3581/\$34.00

Copyright (C) 2016 American Society of Nuclear Cardiology. duration, and count statistics necessitate the use of blurring low-pass filters, resulting in maximum recoverable reconstructed image resolutions rarely better than 10 to $15 \mathrm{~mm}$ FWHM (full-width half maximum) for SPECT, and as a consequence pixel sizes of less than 5 to $7 \mathrm{~mm}$ are rarely used in tomographic images. ${ }^{2}$ The situation is typically better for PET, but not exceedingly so for cardiac PET, particularly if using $\mathrm{Rb}-82$ as a radioisotope.

Suboptimal spatial resolution results in the wellknown partial volume effect, which hides small structures by smearing the activity they contain-a phenomenon that can be actually used to our advantage in gated SPECT and gated PET, where myocardial brightening is taken as a proxy for its thickening from end-diastole to end-systole. ${ }^{3}$ Another way to look at the partial volume effect is to consider how it causes activity to appear in adjacent areas that are not supposed to contain any: this is called the spillover effect, which in small hearts effectively misallocates counts from the myocardium to the left ventricular cavity. This spillover of counts into the myocardial cavity can downright obliterate our ability to visualize it, particularly at endsystole, which in turn results in an overestimation of the left ventricular ejection fraction (LVEF), as pointed out by many investigators in published literature. ${ }^{4-8}$

One of those investigators, Nakajima, is also an author of the study ${ }^{9}$ that is the object of this editorial and is indeed quite familiar with the small heart problem, having conducted and reported on a 106-hospital multicenter trial investigation of gated perfusion SPECT in Japan, where patient body habitus is often considerably different compared to the western world. ${ }^{10}$ Their approach to dealing with small hearts is based on a previously described ${ }^{11}$ commercial software package, modified by a volume-dependent algorithm that shifts the measured LV's endocardial and epicardial surfaces according to a second-degree polynomial equation. The 
shift is inversely proportional to the measured midventricular LV cavity volume, ranging from $3.5 \mathrm{~mm}$ at $0 \mathrm{~mL}$ (effectively "guessing" a cavity where one cannot be seen) to $0 \mathrm{~mm}$ at $85 \mathrm{~mL}$, where spillover is deemed to have abated (see Figure 1 and Ref. 12).

Numerical modeling and compensation of blurring in patients with small hearts have been previously proposed by Case et al in Kansas City and Faber et al at Emory University, although only in abstracts published in 1999-2000, and therefore not explicitly quotable in this editorial. The idea is to devise a correction meant to bring down the overestimated LVEF toward a more physiologically reasonable value, which can theoretically be done if one precisely determines the modulation transfer function (MTF) of the specific imaging system used (camera configuration, acquisition and reconstruction protocol, isotope, collimator, filter, zoom, etc). Yonehama's use of a single equation to produce a "fudge factor" by which to adjust endocardial and epicardial surfaces ${ }^{9}$ is a considerably more simplistic approach, in the sense that it may work on the system for which it was developed, but it cannot be presumed to behave as effectively if the imaging chain changes. In other words, the same small heart that is measured as having an LV cavity volume of $10 \mathrm{~mL}$ when imaged and reconstructed on a given system according to a specific protocol may be measured as 5 or $15 \mathrm{~mL}$ if the system/ protocol changes, with subsequent major changes to the correction applied and to the final LVEF value produced. At the very least, it would be desirable that the second-degree polynomial equation contains some variable relating it to the actual MTF of the imaging chain used.

A more fundamental (not to mention, logical) consideration is that failure to accurately measure the ESV in Figure 1 represents not so much a limitation of the specific quantitative-gated SPECT algorithm used, but rather a suboptimal choice of imaging protocol, given that higher resolution would have been necessary in order to visualize small structures, such as the LV cavity at end-systole.

To the extent that the actual resolution of the acquisition permits, reconstructed resolution can be increased by increasing spatial sampling: if a larger acquisition zoom is used as part of the acquisition protocol, the LV will be magnified, meaning that each pixel in a standard $64 \times 64$ image matrix will represent a smaller portion of the heart. ${ }^{4} \mathrm{~A}$ similar approach requires using a collimator other than the standard parallel-hole type-for example, a hybrid converging/diverging (cardiofocal) collimator, a converging (pinhole) collimator, ${ }^{13}$ or a multi-pinhole system, ${ }^{14}$ the end result being in all cases that the heart fills more of the image matrix. ${ }^{7}$ The fact that zooming in on the LV causes each pixel to contain a lesser number of counts is not as critical of a problem in modern SPECT cameras, since advances in

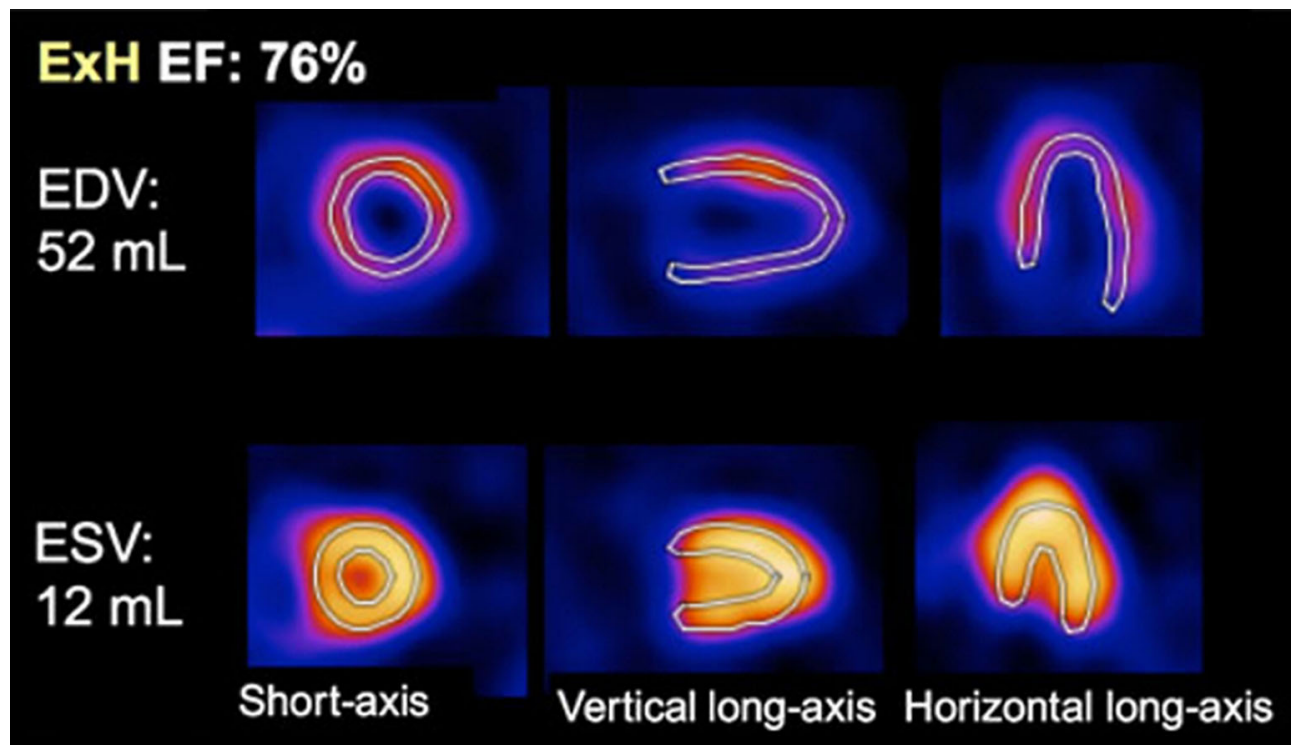

Figure 1. Application of the Exini $(\mathrm{ExH})$ volume-dependent resolution compensation algorithm to a petite 78 yo woman (height $142 \mathrm{~cm} / 4.7^{\prime \prime}$, weight $43 \mathrm{~kg} / 95 \mathrm{lbs}$ ), whose $99 \mathrm{mTc}$-sestamibi-gated tomographic images show apparent visual obliteration of the LV cavity at end-systole. EDV, enddiastolic volume; $E S V$, end-systolic volume; $E F$, ejection fraction. (This figure was originally published in European Journal of Nuclear Medicine and Molecular Imaging under the Creative Commons Attribution 4.0 International License (http://creativecommons.org/licenses/by/4.0/). Nakajima et al. ${ }^{12}$ (C) The Author(s) 2013) 
cardiac hardware and associated acquisition software allow for much higher photon sensitivity and improved image resolution compared to traditional Anger cameras. Even in cardiac PET, resolution recovery methods combined with time-of-flight (TOF) acquisition are now implemented by all vendors, allowing improved contrast, enhanced image resolution, and reduced image noise. ${ }^{15}$

Perhaps the definitive demonstration that standard quantitative cardiac software can do an excellent job even in the smallest hearts, if applied to images acquired with appropriate spatial resolution, can be found in the study by Constantinesco et al, who measured cardiac function in mice using pinhole-gated SPECT and a dedicated smallanimal system. ${ }^{13}$ As evidenced in Figure 2, the exact same clinical quantitative cardiac software, found by Yoneyama to underestimate LV volumes and overestimate LVEFs in pediatric patients, was able to accurately measure reference volumes ranging from 0.01 to $0.1 \mathrm{~mL}$ !

Clearly, using a dedicated, small field-of-view machine for pediatric patients is likely impossible or impractical for most clinics-but employing a higher zoom that makes better use of the image matrix by filling it up with more of the actual heart is eminently feasible, and indeed newer SPECT cameras feature more "cardiocentric', acquisition strategies aimed at exactly that goal. ${ }^{15}$ The fact that zooming in on the heart may truncate thorax contributions at certain angles can be compensated for and is anyway a relatively minor concern when assessing LVEF and myocardial function in general, both of which are less affected by attenuation than perfusion.
The rationale for a specific protocol for pediatric patients include the consideration that a smaller body habitus ought to allow for tighter orbits of rotation for the camera detectors, allowing better geometric capture of the emitted photons and consequently higher count statistics; in addition, scatter (and the blurring it causes) would be expected to be less of an issue with leaner patient bodies.

In our own clinical practice, where most patients are adults with CAD, we do not favor customization of gated SPECT acquisition and/or processing parameters based on individual patients' characteristics and prefer to report LVEFs as being "in the normal range" or "higher than $75 \%$ " whenever overestimation of quantitative LVEF occurs due to small LV size. If pediatric patients were to be imaged with a certain frequency, however, a different approach could be in order. Specifically, the imaging protocol might include a brief planar acquisition followed by the choice of zoom (and/or acquisition orbit) most appropriate for a given patient, based on the observed (or software-determined) heart size. One could even set up multiple acquisition protocols (a standard one and a "pediatric" one, with higher zooms and tighter detector rotation orbits), perhaps to be automatically selected based on patient age, height, and weight, which are typically available in the image header.

Coming back full circle to where we started, aiming before shooting is usually a good idea. If we use a tool calibrated for the average coronary artery disease patient in the western hemisphere to image a 4.7', $95 \mathrm{lbs}$ patient (whether pediatric or not), we cannot expect an

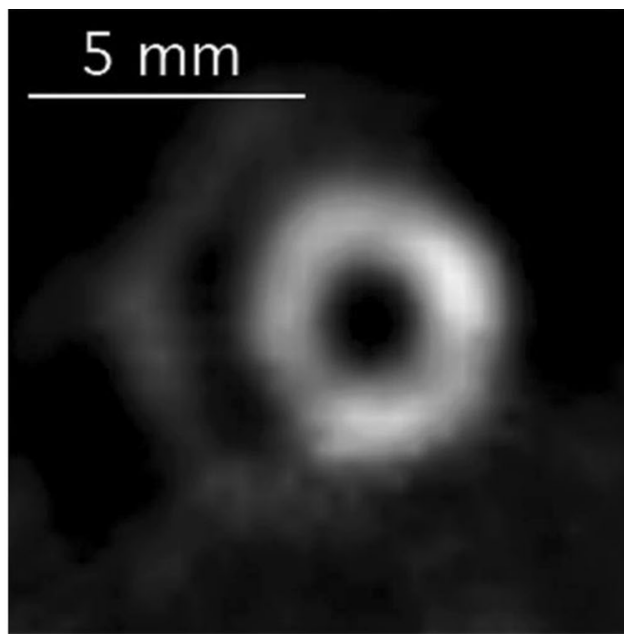

Figure 2. Left Quantitative measurements of reference volumes ranging from 0.01 to $0.1 \mathrm{~mL}$ using a small-animal dedicated pinhole camera and standard automated cardiac software (QGS, CedarsSinai). Right a mid-ventricular, time-summed perfusion short-axis slice of a mouse imaged in-vivo with the same camera. If the imaging system permits good visualization of the left ventricular cavity and surfaces, quantification of volumes and function can be remarkably accurate, with no need for resolution compensation. (This research was originally published in JNM. Constantinesco et al. $^{13}$ (c) by the Society of Nuclear Medicine and Molecular Imaging, Inc) 
accurate result-in a way, it is a bit like performing surgery with a hatchet. Even if we failed to use a sharp enough tool for the job, it does not mean that we should not try to remedy our error, and Yoneyama's report adds to a class of approaches that seek to do that through numerical means. Generally speaking, however, Benjamin Franklin's famous quote that an ounce of prevention is worth a pound of cure probably applies to the small LV in nuclear cardiology, as well as to a great deal of other circumstances.

\section{References}

1. Shannon C. Communication in the presence of noise. Proc Inst Radio Eng 1949;37:10-21. (Reprinted as classic paper in: Proc IEEE, Vol. 86, No. 2, Feb 1998)

2. Cherry S, Sorenson JA, Phelps ME. Physics in nuclear medicine. 3rd ed. Philadelphia: WB Saunders; 2003.

3. Germano G, Erel J, Lewin H, Kavanagh PB, Berman DS. Automatic quantitation of regional myocardial wall motion and thickening from gated technetium-99m sestamibi myocardial perfusion single-photon emission computed tomography. J Am Coll Cardiol 1997;30:1360-7.

4. Nakajima K, Taki J, Higuchi T, Kawano M, Taniguchi M, Maruhashi K, et al. Gated SPET quantification of small hearts: Mathematical simulation and clinical application. Eur J Nucl Med 2000;27:1372-9.

5. Ford PV, Chatziioannou SN, Moore WH, Dhekne RD. Overestimation of the LVEF by quantitative gated SPECT in simulated left ventricles. J Nucl Med 2001;42:454-9.
6. El-Maghraby TAF, El-Rahman NA. Clinical relevance of left ventricular volumes and function assessed by gated SPECT in paediatric patients. Int J Cardiovasc Imaging 2004;20:127-34.

7. Hambye AS, Vervaet A, Dobbeleir A. Variability of left ventricular ejection fraction and volumes with quantitative gated SPECT: Influence of algorithm, pixel size and reconstruction parameters in small and normal-sized hearts. Eur J Nucl Med Mol Imaging 2004;31:1606-13

8. Abe K, Hirakawa K, Yonenaga T, Kobayashi S, Nishimura M, Ayabe Z. Assessment of left ventricular ejection fraction measured by quantitative gated SPECT. Int J Cardiovasc Imaging 2006;22:223-30.

9. Yoneyama H, Nakajima K, Okuda K, Matsuo S, Onoguchi M, Kinuya S, et al. Reducing the small-heart effect in pediatric gated myocardial perfusion single-photon emission computed tomography. J Nucl Cardiol 2016. doi:10.1007/s12350-016-0518-z.

10. Nakajima K, Nishimura T. Inter-institution preference-based variability of ejection fraction and volumes using quantitative gated SPECT with Tc-99m-tetrofosmin: A multicentre study involving 106 hospitals. Eur J Nucl Med Mol Imaging 2006;33:127-33.

11. Lomsky M, Richter J, Johansson L, El-Ali H, Astrom K, Ljungberg $\mathrm{M}$, et al. A new automated method for analysis of gatedSPECT images based on a three-dimensional heart shaped model. Clin Physiol Funct Imaging 2005;25:234-40.

12. Nakajima K, Okuda K, Nystrom K, Richter J, Minarik D, Wakabayashi $\mathrm{H}$, et al. Improved quantification of small hearts for gated myocardial perfusion imaging. Eur J Nucl Med Mol Imaging 2013;40:1163-70.

13. Constantinesco A, Choquet $\mathrm{P}$, Monassier L, Israel-Jost V, Mertz L. Assessment of left ventricular perfusion, volumes, and motion in mice using pinhole gated SPECT. J Nucl Med 2005;46:1005-11.

14. Zeng GL, Stevens AM. Multidivergent-beam stationary cardiac SPECT. Med Phys 2009;36:2860-9.

15. Slomka PJ, Pan T, Berman DS, Germano G. Advances in SPECT and PET Hardware. Prog Cardiovasc Dis 2015;57:566-78. 